\title{
Metrological aspects of researching the specific contact resistivity of ohmic contacts by using the four-contact method
}

\author{
V.N. Sheremet \\ V. Lashkaryov Institute of Semiconductor Physics, NAS of Ukraine, 03028 Kyiv, Ukraine \\ Phone: 38(044) 525-61-82; e-mail:VolodymyrSheremet@gmail.com
}

\begin{abstract}
In this paper, we have considered the four-contact method for measurements of the specific contact resistivity of the ohmic contacts $\left(\rho_{c}\right)$. The presented method for measuring $\rho_{c}$ has been compared with several other methods. Limits of applying this method have been shown.
\end{abstract}

Keywords: ohmic contact, four-contact method, specific contact resistivity.

Manuscript received 19.03.14; revised version received 30.07.14; accepted for publication 29.10.14; published online 10.11.14.

\section{Introduction}

Creation of new semiconductor devices and improvement of existing parameters are impossible without reducing the resistance of the ohmic contacts. The main parameter that characterizes the ohmic contact is the specific contact resistivity $\rho_{c}$. In the case of high resistance of the metal-semiconductor contact, when a device operates, an additional heating occurs in the contact, which negatively impacts on the reliability and efficiency of the device. It is particularly harmful for powerful high-frequency devices. To minimize heating, it is necessary to reduce $\rho_{c}$ of the ohmic contact. With decreasing the $\rho_{c}$ value, the requirements to the accuracy of determining the specific contact resistivity increase. Promising materials for development of high-power microwave devices are semiconductor compounds $\mathrm{A}^{3} \mathrm{~B}^{5}$ such as $\mathrm{GaN}, \mathrm{InN}$, InP, etc. The effective use of these semiconductor materials and increase of the power of microelectronic devices being based on them are impossible without creating reliable heat-resistant ohmic contacts.

\section{Ohmic contact and its resistance}

For measuring the contact resistance, both vertical and planar test structures may be used. In the most complete manner, the existing methods were considered in the work [1]. In response to the direct measurement, there can be obtained a resistance usually presented as the sum of the resistances [2]:

$R=R_{c}+R_{s}+R_{T}+R_{p}+R_{m}$,

where $R$ is the measured resistance, $R_{c}$ - the contact resistance, $R_{s}$ - resistance of semiconductor, $R_{T}$ resistance provoked by current spreading, $R_{p}$ - resistance probes and connecting conductors, $R_{m}$ - resistance of metallization.

Resistance of metallization, probes, connecting conductors, and resistance provoked by current spreading are often small values as compared to the resistance of semiconductor and that of the contact. If the contact resistivity is comparable to the resistance of semiconductor, $R_{T}$ and $R_{p}$ are infinitely small values in relation to $R_{c}$. With further decreasing $\rho_{c}$, account must 
be also taken of the values of the resistance provoked by current spreading and that of semiconductor $R_{p}$.

In the case of a vertical test structure (Fig. 1a), the impact of the current spreading is less than in the case of a planar structure (Fig. 1b), that's why at first, more widely used were the methods using a vertical structure, among which the Cox-Strack method was most widely applied [1]. However, this method is not applicable in the case of contact structures to epitaxial films.

Application of a planar structure to study the specific contact resistivity is preferable because of the reduced number of processes is required to form a test structure. But when using a planar test structure, one should take account of the current spreading that can be characterized by the transfer length $L_{T}-$ the distance from the edge of the contact, near which the current decreases by $e$ times [3-5]. It is determined by the equation [3-5]:

$$
L_{T}=\sqrt{\frac{\rho_{c} t}{\rho_{s}}} .
$$

If the length of contact is longer than the transfer distance, the contact becomes infinite, and its resistance does not depend on the length. Since the entire contact area is not included into the passage of current, it complicates determining the $\rho_{c}$ value. If the contact length is less than the transfer distance, the total resistance is increased as a result of reducing the area of current passage, which must be considered when determining the specific contact resistivity. Accordingly, the effective area of the planar contact becomes less than the actual one.

In the case when the contact length is less than the width of the diffusion region in semiconductor, there observed an effect of the surface current spreading, and the measured total resistance increases to a value determined in the work [2] as follows:
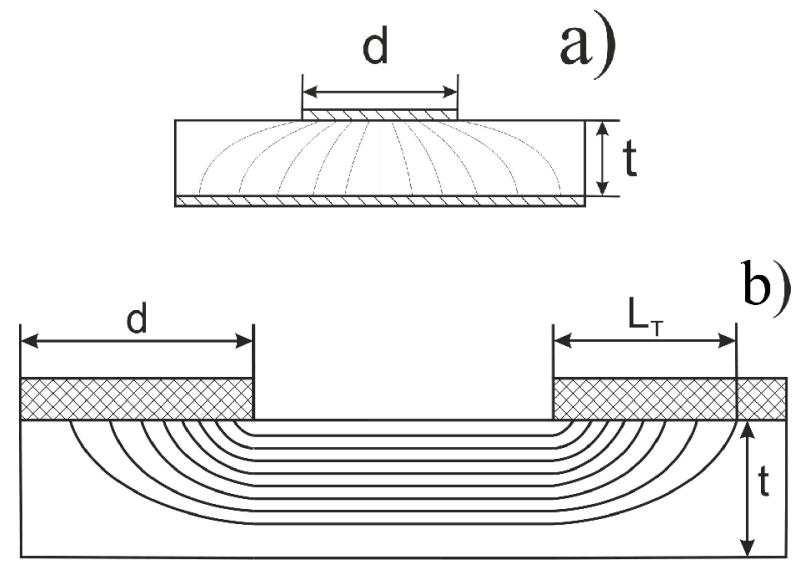

Fig. 1. Current spreading in the vertical (a) and planar (b) test structures ( $d$ is the diameter of contact, $L_{T}-$ transfer length, $t-$ thickness of semiconductor).
$R_{w}=\frac{\rho_{s}}{\pi}\left(\frac{1}{k} \ln \frac{k+1}{k-1}+\ln \frac{k^{2}-1}{k^{2}}\right)$,

where $k=W /(W-w)$ is the correcting parameter, $w-$ contact width, $W$ - width of the diffusion region.

In these methods, both extrapolative and twocontact one, with a planar test structure [5], the resistance caused by current spreading is not taken into consideration, which results in the increased error of measurements. One of the most easy-to-implement planar measurement methods is the four-contact method of $\rho_{c}$ measurements.

\section{Principles of measuring $\rho_{c}$ by using the four-contact method}

The method consists in measuring the voltage at equal distances between the current contacts (Fig. 2) [1]. This method can be used if the transfer length is much larger than the contact size. Also, a necessary condition is $L \gg>r, L \gg t$, where $L$ is the contact length, $t$ - thickness of the semiconductor film. Under these conditions, the contact resistivity is determined from the equation [6]:

$\rho_{c} \approx \pi r^{2}\left\{\frac{V_{1}}{I_{0}}-\left(\frac{V_{2}}{I_{0}}\right) \frac{\ln [(3 L / 2 r)-1 / 2]}{2 \ln 2}\right\}$.

Disadvantage of the method is that it is necessary to fulfill the condition $L>>r$ that implies a significant gap between the contacts, which can result in the inability to determine the contact resistivity at a certain ratio $\rho_{c} / \rho_{s}$.

Due to simplicity of the test structure formation, this method is used for large transfer lengths.

In the case of using the four-contact method, the error of measurements of the specific contact resistivity can be determined using the partial derivative technique:

$$
\begin{aligned}
& \frac{\Delta \rho_{c}}{\rho_{c}} \approx 2 \frac{\Delta r}{r}+\frac{\Delta V}{V}+\frac{\Delta I}{I}+\left(\frac{\Delta L}{L}+\frac{\Delta r}{r}\right) \times \\
& \times \frac{\ln \left(\frac{3}{2} \frac{L}{r}-\frac{1}{2}\right)}{\left(\frac{\rho_{c}}{\rho_{s}}+1\right) 2(3-r)(\ln 2)^{2}-\left(1-\frac{r}{L}\right)\left(\ln \left(\frac{3}{2} \frac{L}{r}-\frac{1}{2}\right)\right)^{2}} .
\end{aligned}
$$

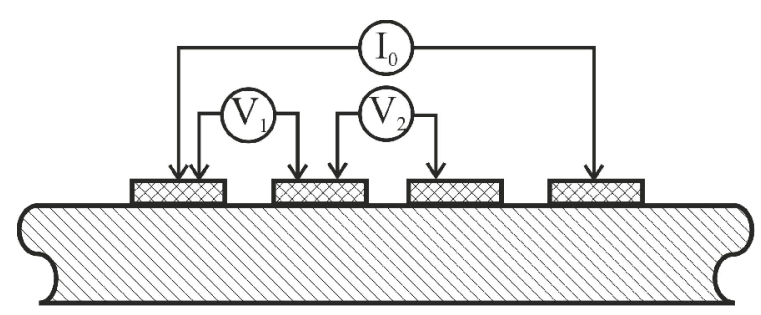

Fig. 2. The principle of measuring the contact resistivity by using the four-contact method. 
The performed calculations have resulted in the dependence of the relative error of the measured contact resistance on the contact radius (Fig. 3). As the obtained dependence illustrates, when increasing the radius of contact and increasing the ratio of the specific contact resistivity to the specific resistance of semiconductor, the measurement error is reduced.

Studied in the work [7] was the effect of microwave treatment on the temperature dependence of the specific contact resistivity of the ohmic contacts $\operatorname{Au}(0.2 \mu \mathrm{m})-$ $\operatorname{TiB}_{\mathrm{x}}(0.1 \mu \mathrm{m})-\mathrm{Ge}(0.04 \mu \mathrm{m})-\mathrm{Au}(0.18 \mu \mathrm{m})-n(2 \mu \mathrm{m})-$ $n^{+}(3 \mu \mathrm{m})-n^{++}(300 \mu \mathrm{m})-\mathrm{InP}$ within the temperature range 100 to $400{ }^{\circ} \mathrm{C}$, which were obtained using the magnetron sputtering of metals and $\mathrm{TiB}_{\mathrm{x}}$ onto the InP surface heated up to $100^{\circ} \mathrm{C}$. The specific contact resistivity was investigated by using the four-contact method before and after microwave treatment for $10 \mathrm{~s}$ at the frequency $2.45 \mathrm{GHz}$ and power density $1.5 \mathrm{~W} / \mathrm{cm}^{2}$. The radius of contacts was equal to $22.5 \mu \mathrm{m}$. Dopant concentration of $n$-InP was $\sim 9 \cdot 10^{15} \mathrm{~cm}^{-3}$ in the $n$-layer, $\sim 5 \cdot 10^{17} \mathrm{~cm}^{-3}$ in the $n^{+}$-layer, $\sim 10^{18} \mathrm{~cm}^{-3}$ in the $n^{++}$. substrate, the thickness of these layers was 2, 3, and $300 \mu \mathrm{m}$, respectively. Ohmic contacts to InP were formed during the RTA at $T=450{ }^{\circ} \mathrm{C}$.

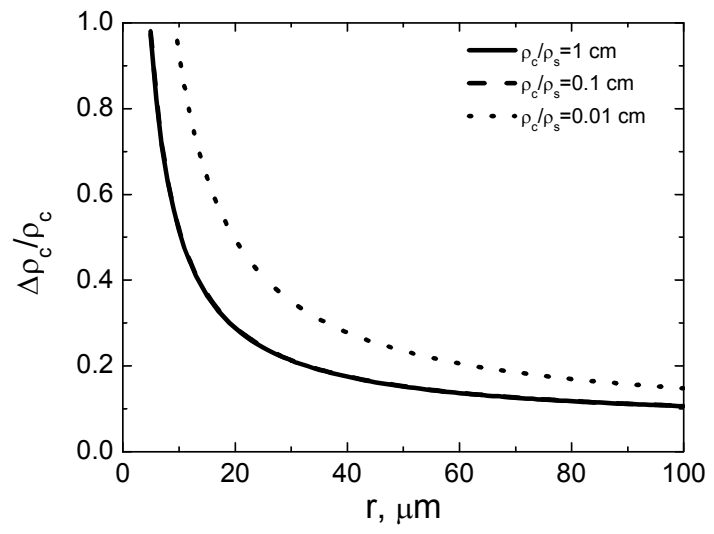

Fig. 3. Dependence of the relative error of measuring $\rho_{c}$ by using the four-contact method on the contact radius for contacts with various ratios $\rho_{c} / \rho_{s}$, under the condition of lithography error $\sim 2.5 \mu \mathrm{m}$.

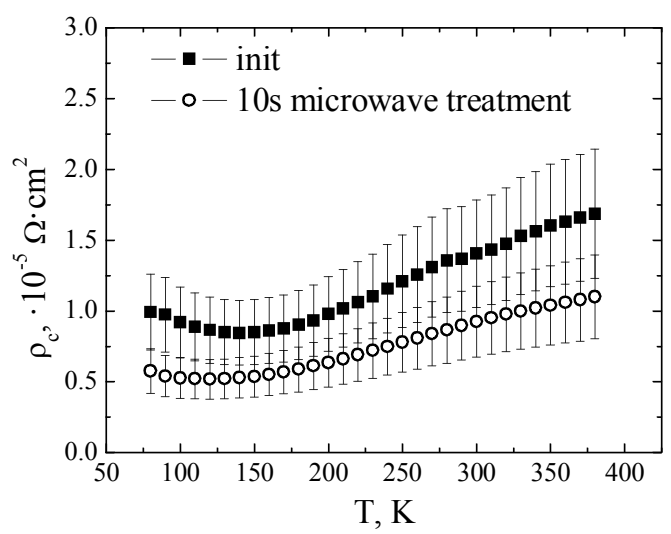

Fig. 4. Dependence of the specific resistivity of ohmic contacts $\mathrm{Au}-\mathrm{TiB} x-\mathrm{Ge}-\mathrm{Au}-n-n^{+}-n^{++}-\mathrm{InP}$ on the temperature before and after microwave treatment for $10 \mathrm{~s}$ [7].
Table. Comparison of relative errors in measurements of the specific contact resistivity obtained using various methods.

\begin{tabular}{|c|c|c|c|c|c|}
\hline Methods & \multicolumn{4}{|c|}{$\Delta \rho_{c} / \rho_{c}$} & $\begin{array}{c}\rho_{s}, \\
\mathrm{Ohm} \cdot \mathrm{cm}\end{array}$ \\
\hline$\rho_{c} / \rho_{s}, \mathrm{~cm}$ & 0.01 & 0.1 & 1 & 10 & \\
\hline Cox-Strack* & 0.88 & 0.27 & 0.08 & - & \\
\hline $\begin{array}{l}\text { LTLM } \\
\left(L<3 L_{T}\right)^{*}\end{array}$ & 0.22 & 0.17 & 0.17 & 0.17 & $10^{-3}$ \\
\hline $\begin{array}{l}\text { LTLM } \\
\left(L>3 L_{T}\right)^{*}\end{array}$ & 0.16 & 0.24 & - & - & $10^{-4}$ \\
\hline CTLM* & 0.27 & 0.15 & 0.08 & 0.05 & \\
\hline Four-contact & 0.14 & 0.10 & 0.10 & - & \\
\hline Kelvin* & 0.25 & 0.15 & 0.05 & 0.04 & \\
\hline $\begin{array}{l}\text { Probing the } \\
\text { interface* }\end{array}$ & 0.14 & 0.07 & 0.04 & 0.02 & \\
\hline
\end{tabular}

Notes. Asterisk * denotes the results obtained in [8], LTLM - linear transmission line method, CTLM - circular transmission line method.

Fig. 4 shows the dependence of $\rho_{c}(T)$ obtained in [7] before and after microwave treatment. As it follows from these curves, within the temperature range 80 to $220 \mathrm{~K}$, the $\rho_{c}$ value changes not exceeding the measurement errors. With the temperature increase, the changes in the specific contact resistivity exceed the error of the measurement method.

\section{Comparison of the measurement methods}

Comparison of errors of measuring the specific contact resistivity was carried out in the work [8]. The errors were compared under the condition of lithography error $\sim 2.5 \mu \mathrm{m}$ close to an error produced in an industrial environment. For the Cox-Strack method, the plate thickness was $400 \mu \mathrm{m}$, for transmission line method (TLM) the semiconductor thickness was $7 \mu \mathrm{m}$, the width of the pads, as for the method of probing the interface, was $1000 \mu \mathrm{m}$.

If the ratio of the specific contact resistivity to the specific semiconductor one is equal to unity, the fourcontact method has a higher error than that in the methods of Cox-Strack, TLM, Kelvin, probing the interface. In the case of reducing the ratio $\rho_{c} / \rho_{\mathrm{s}}$ down to $0.01 \mathrm{~cm}$, the error in measuring the specific contact resistivity takes the minimum values as compared with the abovementioned other methods.

\section{Conclusions}

In this work, we have considered the four-contact method of measuring the specific contact resistivity for planar geometry of pads. The performed calculations 
indicate the promising of using this method for measuring $\rho_{c}$ in the case of the ratio $\rho_{c} / \rho_{\mathrm{s}} \leq 0.01 \mathrm{~cm}$, provided that the requirements for the use of the fourcontact method are fulfilled.

\section{References}

1. D.K. Schroder, Semiconductor Materials and Devices Characterization. Wiley, N.Y. 2006.

2. Chung-Yu Ting, Charles Y. Chen, A study of the contacts of a diffused resistor // Solid State Electron. 14(6), p. 433-438 (1971).

3. H.H. Berger, Contact resistance and contact resistivity // J. Electrochem. Soc. 119(4), p. 507514 (1972).

4. H.H. Berger, Models for contacts to planar devices // Solid State Electron. 15(2), p. 145-158 (1972).
5. H. Murrmann, D. Wiedmann, Current crowding on metal contacts to planar devices // IEEE Trans. Electron. Dev. 16(12), p. 1022-1024 (1969).

6. A.S. Holland, G.K. Reeves, New challenges to the modelling and electrical characterization of ohmic contacts for ULSI devices // Microelectronics Reliability, 40(6), p. 965-971 (2000).

7. A.E. Belyaev, A.V. Sachenko, N.S. Boltovets, V.N. Ivanov, R.V. Konakova, Ya.Ya. Kudryk, L.A. Matveeva, V.V. Milenin, S.V. Novitskii, V.N. Sheremet, Effect of microwave irradiation on resistance of $\mathrm{Au}-\mathrm{TiB} x-\mathrm{Ge}-\mathrm{Au}-n-n^{+}-n^{++}-\mathrm{GaAs}(\mathrm{InP})$ ohmic contacts // Fizika tekhnika poluprovodnikov, 46(4), p. 558-561 (2012), in Russian.

8. V.N. Sheremet, Metrological aspects of measuring the resistance of ohmic contacts // Izvestiya vuzov. Radioelektronika, 53(3), p. 3-12 (2010), in Russian. 